\title{
The Fifth International Conference on Smart City Applications: Preface
}

\author{
Ismail Rakip Karas ${ }^{1}$, Mohamed Ben Ahmed ${ }^{2}$, \\ Anouar Boudhir Abdelhakim², Bernadetta Kwintiana Ane ${ }^{3}$ \\ ${ }^{1}$ Conference Chair, Computer Engineering Department, Karabuk University, Turkey, \\ ismail.karas@karabuk.edu.tr \\ ${ }^{2}$ Conference Co-Chair, Faculte des Sciences et Techniques, Abdelmalek Essaâdi University, \\ Tangier, Morocco,mbenahmed@uae.ac.ma ; aboudhir@uae.ac.ma \\ ${ }^{3}$ Conference Co-Chair, Institute of Computer-aided Product Development Systems, \\ University of Stuttgart, Germany, ane@informatik.uni-stuttgart.de
}

This Conference Proceedings volume contains the written versions of the contributions presented during the $5^{\text {th }}$ International Conference on Smart City Applications.

At first, the event had been planned to organized in Safranbolu Campus of Karabuk University, Türkiye. Then, it has been converted to the online conference because of the Covid-19 situation. It took place with the motto of "Virtual Safranbolu" by inspiring historical UNESCO Heritage city Safranbolu, on October 07-08, 2020. The conference provided a setting for discussing recent developments in a wide variety of topics including Geo-Smart Information Systems, Smart Cities, 3D City Modeling and Visualization, Smart Building and Home Automation, Smart Environment and Smart Agriculture, Location Based Services, GeoInformation for Mobile, Wearable Technologies and Wireless Sensor Networks, Building Information Modeling, Virtual and Augmented Reality, Big Data and Urban Data Analytics, Smart Healthcare, Smart Economy and Digital Business, Smart Education and Intelligent Learning System, and etc.

The event has been a good opportunity for the more than 300 participants coming from more than 30 countries of the world to present and discuss topics in their respective research areas. In addition, four keynote speakers presented latest achievements on their fields; Alias Abdul Rahman "Smart Cities and Geo-Spatial Technologies", Şule Erten Ela "Smart Cities and Energy", Bülent Bayram "Deep learning applications for shoreline extraction from Landsat and Sentinel satellite Imagery", Attaullah Shah "Review Use of Modern Technologies in Creating Smart Cities".

The 63 papers that were selected as a result of double-blind review process and presented during the conference were accepted for the final publication in the ISPRS Archives.

We would like to thank all participants, organizing and scientific committee members, and session chairs for their contributions to the conference program and these Proceedings. 


\section{CONFERENCE CHAIR}

İsmail Rakıp Karaș, Karabuk University, Turkey www.ismailkaras.com

\section{CONFERENCE CO-CHAIRS}

Mohamed Ben Ahmed, FST, Tangier UAE University, Morocco

Anouar Boudhir Abdelhakim, FST, Tangier UAE University, Morocco

Bernadetta Kwintiana Ane, University of Stuttgart, Germany

\section{STEERING COMMITTEE}

İsmail Rakıp Karaș, Karabuk University, Turkey

Alias Abdul Rahman, Universiti Teknologi Malaysia

Mohamed Wahbi, EHTP Casablanca, Morocco

Mohammed Bouhorma, FST, Tangier UAE University, Morocco

Chaker El Amrani, FST, Tangier UAE University, Morocco

Bernard Dousset, UPS, Toulouse, France

Rachid Saadane, EHTP Casablanca, Morocco

Ali Youness, FS, Tetouan, Morocco

Alaoui Abdellaoui El Arbi, EIGSI Casablanca, Morocco

\section{ORGANIZING COMMITTEE}

Ben Ahmed Mohamed, FST Tangier, Morocco

Olga Sergeyeva, Saint-Petersburg University, Russia.

Wassila Mtalaa, Luxembourg Institute of Science and Technology, Luxembourg

Domingos Santos, Polytechnic Institute Castelo Branco, Portugal

Joel Rodrigues, National Institute of Telecommunications , Brazil

Luís Braganca, University of Minho, Portugal

Bernadetta Kwintiana Ane, University of Stuttgart, Germany

Senthil Kumar, Hindustan College of Arts and Science, India

Amine El Haddadi, Paul Sabatier University, Toulouse, France

\section{LOCAL ORGANIZING COMMITTEE}

Idris Kahraman, Karabuk University

Emrullah Demiral, Karabuk University

Mustafa Aksin, Karabuk University

Kadriye Oz, Karabuk University

Hacer Kübra Köse, Sinop University

Berna Gunes, Karabuk University

Umit Atila, Karabuk University

Kasim Ozacar, Karabuk University

Yasin Ortakci, Karabuk University

Muhammed Kamil Turan, Karabuk University

Sohaib Abujayyab, Karabuk University, Turkey

Emre Yücer, Karabuk University 


\section{SCIENTIFIC COMMITTEE}

Ismail Rakip Karas, Karabuk University, Türkiye

Abdel-Badeeh M. Salem, Ain Shams University, Egypt

Abderrahim Ghadi, FSTT UAE, Morocco

Abdullah Elen, Bandirma Onyedi Eylül University, Türkiye

Abdullah Emin Akay, Bursa Technical University, Türkiye

Abdurrahman Geymen, Erciyes University, Türkiye

Accorsi, Riccardo, Bologna University, Italy

Adib Habbal, Karabuk University, Türkiye

Adnan Alajeeli, Karabuk University, Türkiye

Aftab Ahmed Khan, Karakoram International University, Pakistan

Ahmad S. Almogren, King Saud University, Saudi Arabia

Ahmed Kadhim Hussein, Babylon University, Iraq

Alabdulkarim Lamya,King Saud University,Saudi Arabia

Alghamdi Jarallah, Prince Sultan University, Saudi Arabia

Ali Jamali, Universiti Teknologi Malaysia

Alias Abdul Rahman, Universiti Teknologi Malaysia

Anabtawi Mahasen, Al-Quds University, Palestine

Anton Yudhana, Universitas Ahmad Dahlan, Indonesia

Arif Çağdaş Aydinoglu, Gebze Technical University, Türkiye

Arioua Mounir UAE, Morocco

Assaghir Zainab , Lebanese University, Lebanon

Astitou Abdelali, UAE, Morocco

Aydın Üstün, Kocaeli University, Türkiye

Aziz Mahboub, FSTT UAE, Morocco

Bahadır Ergun, Gebze Technical University, Türkiye

Barış Kazar, Oracle, USA

Bataev Vladimir, Zaz Ventures, Switzerland

Behnam Alizadehashrafi, Tabriz Islamic Art University, Iran

Behnam Atazadeh, University of Melbourne, Australia

Ben Yahya Sadok, Faculty of Sciences of Tunis, Tunisia

Bessai-Mechmach Fatma Zohra, CERIST, Algeria,

Biswajeet Pradhan, University of Technology Sydney, Australia

Berk Anbaroğlu, Hacettepe University, Türkiye

Bolulmalf Mohammed, UIR, Morocco

Boutejdar Ahmed, German Research Foundation, Bonn, Germany

Burhan Selcuk, Karabuk University, Türkiye

Bulent Bayram, Yildiz Technical University, Türkiye

Caner Ozcan, Karabuk University, Türkiye

Caner Güney, Istanbul Technical University, Türkiye

Chadli Lala Saadia, University Sultan Moulay Slimane, Morocco

Cumhur Şahin, Gebze Technical University, Türkiye

Damir Žarko, Zagreb University, Croatia

Dominique Groux, UPJV, France

Dousset Bernard UPS, Toulouse, France

Edward Duncan, The University of Mines \& Technology, Ghana

Eehab Hamzi Hijazi, An-Najah University, Palestine

E1 Kafhali Said, Hassan 1st University, Settat, Morocco

Eftal Şehirli, Karabuk University, Türkiye

El Malahi Mostafa, USMBA University, Fez, Morocco 
El Mhouti Abderrahim, FST, Al-Hoceima, Morocco

El Haddadi Anass, UAE University, Morocco

El Hebeary Mohamed Rashad, Cairo University, Egypt

El Ouarghi Hossain, ENSAH UAE University, Morocco

Elif Sertel, Istanbul Technical University, Türkiye

Emre Yücer, Karabuk University, Türkiye

Emrullah SONUÇ, Karabuk University, Türkiye

En-Naimi El Mokhtar, UAE, Morocco

Enrique Arias Castilla-La Mancha University, Spain

Tolga Ensari, Istanbul University, Türkiye

Ferhat Atasoy, Karabuk University, Türkiye

Filip Biljecki, National University of Singapore

Francesc Anton Castro, Technical University of Denmark

Ghulam Ali Mallah, Shah Abdullatif University, Pakistan

Habibullah Abbasi, University of Sindh, Pakistan

Haddadi Kamel Iemn, Lille University, France

Hakan Kutucu, Karabuk University, Türkiye

Hanane Reddad, USMS University, Morroco

Hazim Tawfik, Cairo University,Egypt

Huseyin Bayraktar, General Directorate of GIS, Türkiye

Hüseyin Pehlivan, Gebze Technical University, Türkiye

Huseyin Topan, Bulent Ecevit University, Türkiye

Huseyin Zahit Selvi, Konya Necmettin Erbakan University

İlhami Muharrem Orak, Karabuk University, Türkiye

Ilker Türker, Karabuk University, Türkiye

Iman Elawady, Ecole Nationale Polytechnique d'Oran, Algeria

Indubhushan Patnaikuni, RMIT - Royal Melbourne Institute of Technology, Australia

Ismail Büyüksalih, Bimtaş A.Ş., Türkiye

Ivin Amri Musliman, Universiti Teknologi Malaysia

J. Amudhavel, VIT Bhopal University, Madhya Pradesh, India

Jaime Lioret Mauri Polytechnic University of Valencia, Spain

Jus Kocijan, Nova Gorica University, Slovenia

Kadir Ulutaş, Karabuk University

Kasım Ozacar, Karabuk University

Khoudeir Majdi Iut, Poitiers university, France

Labib Arafeh, Al-Quds University, Palestine

Laila Moussaid, ENSEM, Casablanca, Morocco

Lalam Mustapha, Mouloud Mammeri University of Tizi Ouzou, Algeria

Loncaric Sven, Zagreb University, Croatia

Lotfi Elaachak, FSTT, UAE, Morocco

Mademlis Christos, Aristotle University of Thessaloniki, Greece

Mehmet Akbaba, Karabuk University, Türkiye

Mete Celik, Erciyes University, Türkiye

Miranda Serge, Nice University, France

Mohamed El Ghami, University of Bergen, Norway

Mohammad Sharifikia, Tarbiat Modares University, Iran

Mousannif Hajar, Cadi Ayyad University, Morocco

Mufit Cetin, Yalova University, Türkiye

Muhamad Uznir Ujang, Universiti Teknologi Malaysia

Muhammad Imzan Hassan, Universiti Teknologi Malaysia 
Muhammed Kamil Turan, Karabuk University, Türkiye

Murat Yakar, Mersin University, Türkiye

Murat Lüy, Kırıkkale University, Türkiye

Mustafa Akgul, Istanbul University, Türkiye

My Lahcen Hasnaoui, Moulay Ismail University, Morocco

Mykola Kozlenko, Vasyl Stefanyk Precarpathian National University, Ukraine

Nafil Khalid, UM5, Morocco

Nesrin Aydin Atasoy, Karabuk University, Türkiye

Nusret Demir, Akdeniz University, Türkiye

Oğuz Fındık, Karabuk University, Türkiye

Oğuzhan Menemencioğlu, Karabuk University, Türkiye

Omar Dakkak, Karabuk University, Türkiye

Omer Muhammet Soysal, Southeastern Louisiana University, USA

Ouederni Meriem ,INP - ENSEEIHT Toulouse, France

R. S. Ajin, DEOC DDMA, Kerala, India

Rani El Meouche, Ecole Spéciale des Travaux Publics, France

Raif Bayır, Karabuk University, Türkiye

Rafet Durgut, Karabuk University, Türkiye

Saffet Erdogan, Harran University, Türkiye

Sagahyroon Assim, American University of Sharjah, United Arab Emirates

Saied Pirasteh, University of Waterloo, Canada

Savas Durduran, Konya Necmettin Erbakan University, Türkiye

Sedat Bakici, Turkish Cadastre Office, Türkiye

Sibel Senan, Istanbul University, Türkiye

Senthil Kumar, Hindustan College of Arts and Science, India

Serdar Bayburt, Bimtaş A.Ş., Türkiye

Seyit Ali Kayış, Karabuk University, Türkiye

Siddique Ullah Baig, COMSATS Institute of Information Technology, Pakistan

Slimani Yahya, Manouba University, Tunisia

Sohaib Abujayyab, Karabuk University, Türkiye

Sonja Grgić, Zagreb University, Croatia

Sri Winiarti, Universitas Ahmad Dahlan, Indonesia

Suhaibah Azri, Universiti Teknologi Malaysia

Sunardi, Universitas Ahmad Dahlan, Indonesia

Sule Erten Ela, Ege University, Türkiye

Tebibel Bouabana Thouraya, ESI, Alger, Algeria

Umit Atila, Karabuk University, Türkiye

Umit Isikdag, Mimar Sinan Fine Arts University, Türkiye

Umran Koylu, Erciyes University, Türkiye

Xiaoguang Yue, International Engineering and Technology Institute, Hong Kong

Yasin Ortakc1, Karabuk University, Türkiye

Yasyn Elyusufi, FSTT, UAE, Morocco

Yüksel Çelik, Karabuk University, Türkiye

Youness Dehbi, University of Bonn, Germany

Yusuf Arayıc1, Northumbria University, UK

Yusuf Yarg1 Baydilli, Karabuk University, Türkiye

Zafer Albayrak, Karabuk University, Türkiye

Zennure Uçar, Düzce University, Türkiye

Zigh Ehlem Slimane, INTTIC, Oran, Algeria

Zouhri Amal, USMBA University, Fez, Morocco 